\title{
A Rare Case: Tuberculous Peritonitis, Encapsulating Peritoneal Sclerosis, and Incisional Hernia in Continuous Ambulatory Peritoneal Dialysis Patient
}

\author{
Enita R. Kurniaatmaja ${ }^{1}$, Ria Bandiara ${ }^{2}$ (D) Ika Kustiyah Oktaviyanti ${ }^{3}$, Mohammad Rudiansyah ${ }^{1 *}$ (D) \\ ${ }^{1}$ Department of Internal Medicine, Division of Nephrology and Hypertension, Faculty of Medicine, Universitas Lambung \\ Mangkurat, Ulin General Hospital, Banjarmasin, Indonesia; ${ }^{2}$ Department of Internal Medicine, Division of Nephrology and \\ Hypertension, Faculty of Medicine, Universitas Padjadjaran, Hasan Sadikin Hospital, Bandung, Indonesia; ${ }^{3}$ Department of \\ Anatomy Pathology, Faculty of Medicine, Universitas Lambung Mangkurat, Ulin General Hospital, Banjarmasin, Indonesia
}

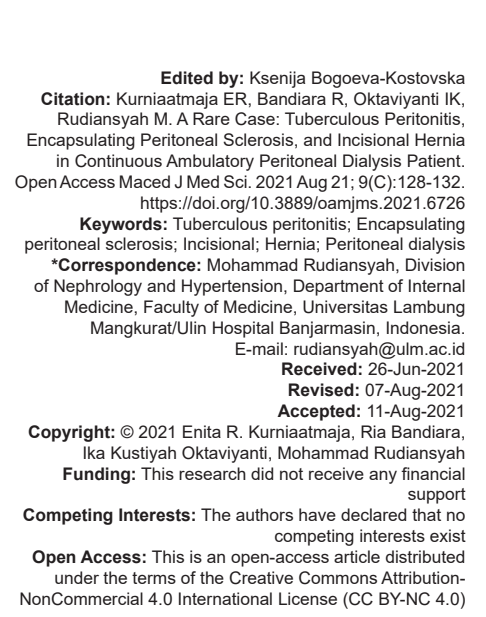

\section{Introduction}

Peritonitis is perceived as one of the most common infectious complications in peritoneal dialysis (PD) with estimated frequency of 1: 20-30 patients per month and mortality rate of $15-18 \%$ [1]. The condition is possibly caused by mycobacteria, although in rare proportion (<3\%). However, countries in Asia and Africa show a higher prevalence, but not all the infections are due to Mycobacteria tuberculosis (Mtb). Therefore, specific tests are needed for proper diagnosis [2].

Similarly, encapsulating peritoneal sclerosis (EPS), an uncommon complication of PD with incident rate varying from 0.7 to 13.6 per 1000 patients per year. The condition causes ultrafiltration failure and intestinal obstruction, although in rare cases, and is associated with significant morbidity and high mortality [3].

This case is further discussed by a patient with tuberculous encapsulating peritonitis, EPS, and incisional hernia. The importance of the subject matter is due to an uncommon occurrence, termed the presence of multiple complications in end-stage kidney disease patients undergoing continuous ambulatory PD (CAPD) and requires comprehensive management.

\section{Case Illustration}

A male, 37 years old, arrived the Emergency Room at Ulin Hospital Banjarmasin, Indonesia, with severe recurrent abdominal pain, but then extended beyond the region the next day. Consequently, complaints are accompanied by a change in the PD fluid to cloudy coloration (Figure 1), followed by nausea, and brownish vomiting up to 5 times. However, within 2 months before hospital admission, the fluid replacement became less effective leading to the 


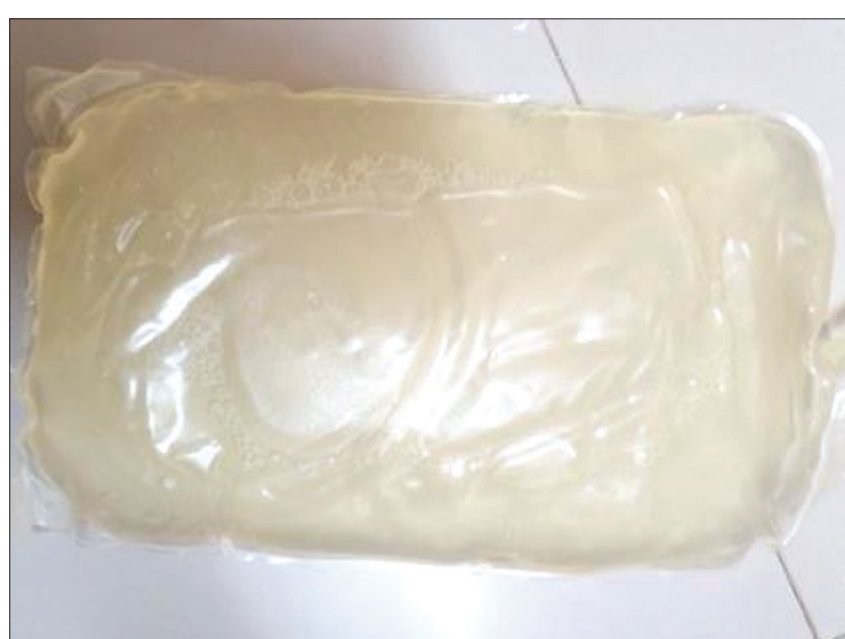

Figure 1: Continuous ambulatory peritoneal dialysis fluid

swelling of both lower legs. This also resulted to a soft, intermittent bulge in the surgical suture area expanding into the abdominal cavity after filling the dialysate and decreases with subsequent drying. The bump is clearly visible as the patient sits or stands but disappears at lying down. Further observations include less appetite, often nauseous, defecates less smoothly, and hard sensation on the lower right abdomen, as well as a $7 \mathrm{~kg}$ weight loss in the last 5 months. There was also 2-year history of hemodialysis (HD) with a switch to CAPD for a year. During PD, the patient suffered peritonitis for the $3^{\text {rd }}$ time, with the latest encountered 4 months ago.

Based on physical examination, general condition was moderate, compos mentis, with blood pressure $130 / 90 \mathrm{mmHg}$, pulse 114 times/min, breath rate 24 times $/ \mathrm{min}$, and body temperature $36.7^{\circ} \mathrm{C}$. In addition, the head and neck revealed anemic conjunctiva. On asymmetric thoracic assessment, the right lung breath declined from intercostal space $\mathrm{VI}$ downward accompanied by dim percussion. Abdominal inspection reported a parallel surface of the abdominal wall to the chest enclosure, PD catheter (+), and a $5 \mathrm{~cm}$ long surgical wound in the linea mediana infraumbilical at a sitting position showed a soft bulge but disappeared on lying down (Figure 2). However, no erythema and exudate were discovered around the PD catheter exit site, although, auscultation highlighted a decline in bowel sounds. On palpation, tenderness was observed in all abdomen areas, while on the extremities, pitting edema was in both legs (Figure 3 ).

The initial laboratory results confirmed anemia (Hemoglobin $7.6 \mathrm{~g} / \mathrm{dl}$ ), and the abdominal radiology analysis of 3 positions showed chest pleural effusion and suspected ascites, but no cardiomegaly.

Based on dialysate fluid analysis (Table 1), cloudy white effluent and Ziehl-Neelsen (ZN) +1 staining were obtained and then confirmed by checking the dialysate fluid acid-resistant bacteria (ARB) to achieve +3. Furthermore, the GeneXpert MTB/RIF examination of dialysate fluid Mtb detected, rifampicin

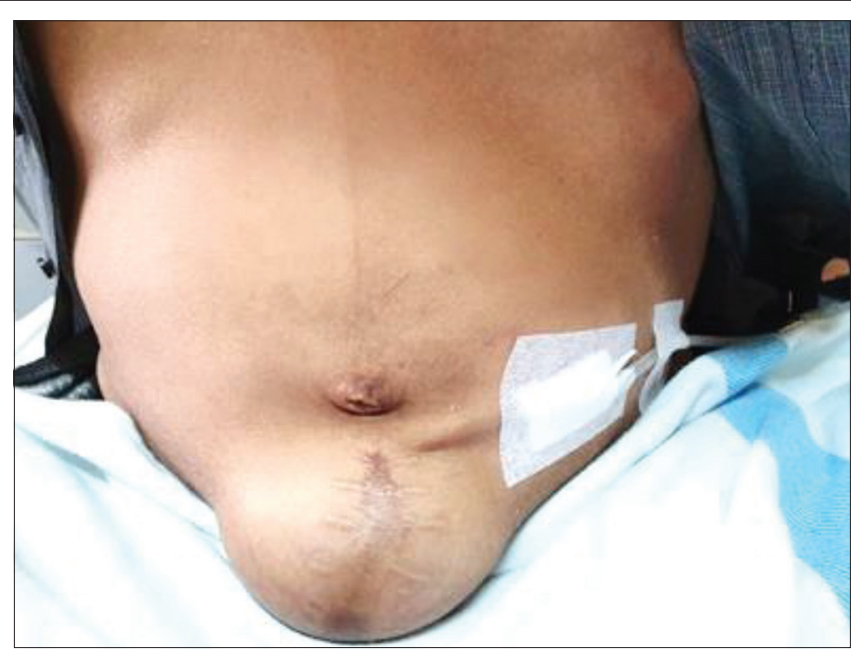

Figure 2: Incisional hernia

sensitive, although, the culture did not record any bacterial growth. Pleural fluid analysis results suggest a transudate, with no bacterial growth (Table 2).

An abdominal computed tomography (CT) scan, without contrast, revealed chronic processing of both kidneys with ascites, therefore supporting sclerosing encapsulating peritonitis (SEP) underlying PD.

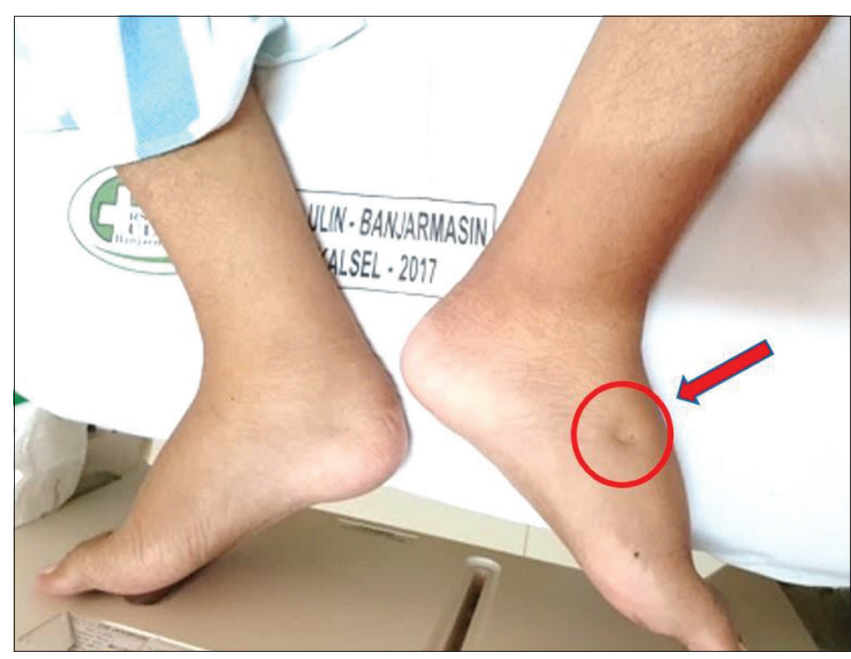

Figure 3: Leg edema

\section{Discussion}

Tuberculous peritonitis is one of the most common infectious complications in PD (DP) with estimated frequency of 1: $20-30$ patients per month and a mortality rate of $15-18 \%$ [1]. Clinical symptoms include cloudy dialysate fluid, abdominal pain, fever, constipation, and diarrhea. In addition, proper diagnosis is achievable based on at least two of the following criteria: (1) Abdominal pain with or without cloudy dialysis effluent, (2) calculated dialysate cells $>100 / \mu \mathrm{L}$ or $>0.1 \times 10^{9 / \mathrm{L}}$ (after a residence time of 
Table 1: Dialysate fluid analysis

\begin{tabular}{llll}
\hline Variables & $13 / 8 / 19$ & $19 / 8 / 19$ & $20 / 8 / 19$ \\
\hline Glucose & 655 & 641 & \\
Lactate dehydrogenase & 127 & 190 & \\
Albumin & 0.4 & 0.4 & \\
Color & White & No color & \\
Clarity & Cloudy & Cloudy & \\
Smell & Odorless & Odorless & \\
Gram stain & Not found & Not found \\
Ziehl-Neelsen stain & Positive & Positive \\
Erythrocyte & 0.001 & 0.001 & \\
Leukocyte & 278 & 301 & \\
Polymorphonuclear & 87 & 82 & \\
Mononuclear & 13 & 18 & \\
Acid-resistant bacteria stain & \multicolumn{4}{l}{ Mtb detected, rifampicin sensitive } \\
GeneXpert MTB/RIF & \multicolumn{3}{l}{} \\
\hline${ }^{*}$ MTB: Mycobacteria tuberculosis, RIF: Rifampicin.
\end{tabular}

at least $2 \mathrm{~h}$ ), with the number of polymorph nuclear cells $>50 \%$, and (3) obtained a positive dialysate culture [4].

Table 2: Pleural fluid analysis

\begin{tabular}{ll}
\hline Variables & Results \\
\hline Glucose & 43 \\
Lactate dehydrogenase & 273 \\
Albumin & 1.3 \\
Color & Yellow \\
Clarity & Cloudy \\
Smell & Odorless \\
Clot & Positive \\
Gram stain & Not found \\
Ziehl-Neelsen stain & Negative \\
Erythrocyte & 0.001 \\
Leukocytes & 21 \\
Polymorphonuclear & $14 \%$ \\
Mononuclear & $86 \%$ \\
\hline
\end{tabular}

In this case, a complaint of abdominal pain was involved accompanied by a change in the dialysate fluid to cloudy coloration. This observation does not necessarily reflect peritonitis, but any cloudy effluent is treated as a potential suspect, despite other differential diagnoses. However, a solid confirmation is dependent on the examination of cell counts and peritoneal fluid culture. Based on peritoneal fluid analysis, the dialysis effluent changed to cloudy white, with a cell count of 278 , therefore peritonitis diagnosis is successful [2].

In rare situation $(<3 \%)$, peritonitis is also caused by mycobacteria, but not all the infections are due to Mtb. However, specific tests are needed for complete diagnosis [2]. Suspicion of tuberculous peritonitis is considered in all cases initially identified with bacterial peritonitis, but certain symptoms persist after conventional antibiotic therapy. Therefore, proper examination is deliberated in any patient with refractory peritonitis or relapse with negative bacterial cultures [5], [6].

The results of initial analysis showed ZN stain +1 and then confirmed by checking the ARB stain dialysate fluid to achieved +3 . Furthermore, GeneXpert MTB/RIF examination of dialysate fluid Mtb detected, rifampicin sensitive, but the culture did not show any bacterial growth.

There are various tests for definitive diagnosis of TB peritonitis, ranging from microscopic examination (Ziehl-Neelsen and auramine-rhodamine stains), solid media culture, including Lowenstein-Jensen, liquid culture on Middlebrook broth, Mycobacteria growth indicator tube, polymerase chain reaction (PCR) for gene IS6110, line probe test (Hain Life science, Germany), real-time PCR using single nucleotide polymorphisms, to GeneXpert MTB (Mtb)/RIF (rifampicin resistance) assay [2].

In this case, ZN test was positive and the technique was less sensitive, without the ability to detect resistance. Therefore, with a positive AFB result, further investigation of the peritoneal fluid with GeneXpert MTB/RIF, where the patient showed a positive result, is needed. Moreover, the GeneXpert MTB/RIF examination is known to improve the ability to diagnose TB (pulmonary and extrapulmonary), as well as additional information on rifampin resistance [2].

The presentation of peritoneal TB tends to vary between DP and HD patients, where the occurrence in DP appeared in the form of peritonitis without improvement, while as ascites in HD. In this case, the possibility of TB preceded by pulmonary tuberculosis has been considered, and the results of a thorax X-ray examination indicated a right pleural effusion [7]. However, after confirmation through an effusion fluid analysis and culture assessment, negative evaluations were obtained. Therefore, based on all the supporting data above, a diagnosis of tuberculous peritonitis was validated.

EPS is a rare complication of $P D$, characterized by fibrosis and intraperitoneal inflammation leading to the enlargement of the bowel loops. However, the incidence varies between 0.7 and 13.6 per 1000 patients per year and possibly causes ultrafiltration failure and intestinal obstruction in association with significant morbidity and high mortality [3].

There are two types of EPS, primary, and secondary. The primary is caused by idiopathic while the secondary is often associated with abdominal tuberculosis, use of beta-blockers (Practolol), PD, liver cirrhosis, sarcoidosis, systemic lupus erythematosus, ventriculoperitoneal and peritoneovenous shunt, abdominal surgery history, intraperitoneal chemotherapy, endometriosis, and gastrointestinal malignancies [8].

The major risk factors for EPS are PD duration and recurrent peritonitis. Meanwhile, other conditions, including the use of specific dialysate fluids (of higher glucose concentration, use of acetate as a dialysate buffer, and bio-incompatible), drugs, e.g., beta-blockers and calcineurin inhibitors, as well as decreased ultrafiltration and increased dissolved substance transport are considered possible risk factors [2].

Furthermore, EPS is suspected in patients known to have experienced PD and with specific gastrointestinal symptoms (e.g., abdominal pain, nausea, loss of appetite, constipation, diarrhea, and vomiting) without a clear explanation, reduced ultrafiltration ability, therefore resulting in fluid retention and edema. 
Definitive diagnosis is confirmed by laparotomy and/or laparoscopy, indicating a characteristic peritoneum thickening to envelop intestinal contents. However, CT scan is referred as a possible alternative and the results showed calcification of the peritoneum, thickening, and intestine dilation. Intestinal thickening and peritoneal calcification are the most specific features, accompanied by characteristic signs and symptoms [3].

In this case, complaints of nausea, vomiting, decreased appetite, and constipation without improvement after receiving prokinetic therapy and laxatives were common. This was further confirmed by an abdominal CT scan, and the result revealing a thickening of the peritoneum, partly with calcification, air-filled intestinal, dilated colon with wall thickening. The mesentery lining of the liver and intestine appears thick with multiple calcifications (Figure 4) supporting SEP underlying PD. Based on the clinical symptoms and the above support, EPS diagnoses are concluded.

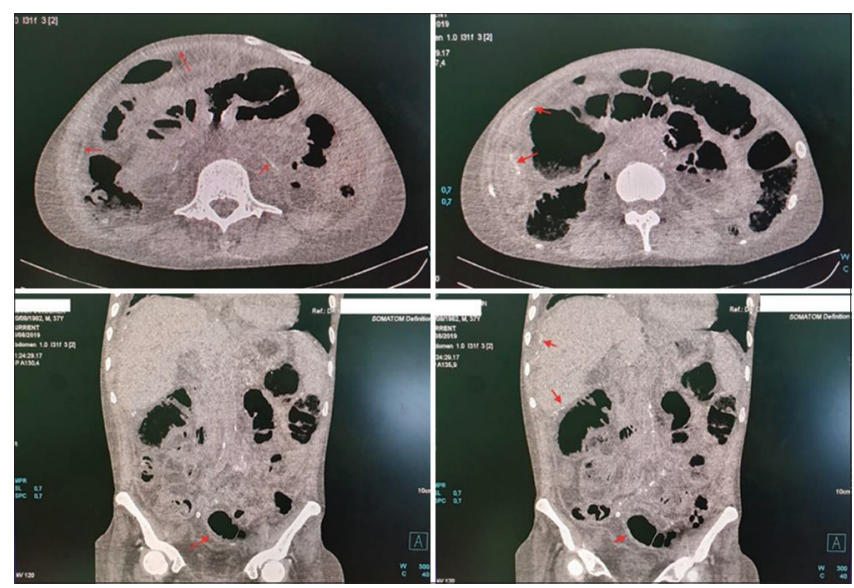

Figure 4: The abdominal computed tomography scan without contrast, peritoneal thickening, and calcification (red arrow)

Tuberculous peritonitis treatment protocols are probably based on general practices but are often initiated with 4 drugs, termed rifampin, isoniazid, pyrazinamide, and ethambutol. Isoniazid and rifampin are administered for 12-18 months, along with pyrazinamide and ofloxacin in the first 3 months. Oral pyridoxine (50-100 mg) is dispensed daily to avoid isoniazid-induced neurotoxicity. Ethambutol is associated with a high risk of optic neuritis in dialysis patients therefore is employed with appropriate dose reductions [9].

In this case, the patient received a category I antituberculosis drug therapy (OAT), termed 2 months isoniazid, rifampicin, ethambutol, and pyrazinamide followed by 4 months of isoniazid and rifampin, as both were subsequently eliminated by biliary excretion, therefore no dose adjustment was required. However, patients with both renal impairment and kidney failure were administered pyridoxine to prevent peripheral neuropathy while receiving isoniazid. The excretion of ethambutol and pyrazinamide metabolites occurs in the kidneys, therefore an adjustment in the dose of both drugs is required. Ethambutol is assigned $15 \mathrm{mg} / \mathrm{kg}$ body weight (BW) and pyrazinamide $25 \mathrm{mg} / \mathrm{kgBW}$ at 3 times per week [10], [11].

The EPS management comprised of medical therapy with tamoxifen and resting the peritoneum (temporary switch to HD). Furthermore, special attention is needed on the nutritional status as well as supportive care [12]. Tamoxifen is dispensed at a dose of $10 \mathrm{mg} 3$ times a day for a minimum of 3 months, combined with daily prednisone $40 \mathrm{mg}$ for a minimum of 4 months, then tapered for the next 6-8 weeks.

An incisional hernia is a tissue lump formed at the site of the surgical scar healing. This category accounts for $15-20 \%$ of all abdominal hernias and tends to occur in any cut in the abdomen (in the median, paramedian, subcostal, McBurney, Pfannenstiel, and flank) depending on the location and size [13].

Midline openings have the highest occurrence (3-20\%), and in a systematic review, the risk of an incisional hernia was higher compared to the transverse (relative risk [RR]: $1.77,95 \%$ confidence interval $[\mathrm{Cl}]$ : 1.09-2.87), and paramedian classes (RR: 3.41, 95\% Cl: 1.02-11.45) [13]. The vertical cut showed greater risk of hernia than the transverse/oblique, and the upper abdominal incision is more prone to hernia compared to the lower situation. The risk factors for an incisional hernia include patient (e.g., old age, obesity, smoking, malnutrition, immunosuppressive therapy, connective tissue disorders) and technical (wound infection, suboptimal fascia closure, and type of abdominal surgery).

In patients undergoing PD, the presence of hernia influences the dialysis clearance and ultrafiltration, including incisional hernias [14]. A low body mass index accompanied by muscle mass wasting and polycystic kidney disease are suspected risk factors, including increased intra-abdominal pressure, although no strong evidence exists. In all the above-mentioned intentions, corrective surgical intervention is required.

In this case, a soft bulge on the lower abdominal wall is observed by inspection, denoting an incisional hernia in the healing area of the surgical scar. The patient complained of this condition for the last 2 months, and the size of the protrusion increases after filling the dialysate into the peritoneal cavity and decreases after draining. This protrusion is clearly visible as the patient sits or stands but disappears on lying down. Furthermore, there was no visible external leakage of the dialysate along the scar, clearly indicating an incisional hernia condition.

In this case, considering several complications associated with $\mathrm{PD}$, ranging from recurrent peritonitis, tuberculous peritonitis, EPS, and incisional hernia responsible for ineffective PD ultrafiltration, CAPD was discontinued and switched to renal replacement therapy to HD twice a week, as well as installing temporary 
double lumen catheter access. In addition to receiving category I antituberculous drug therapy and prednisone $40 \mathrm{mg}$ per day, herniorrhaphy surgery and Tenckhoff catheter were also performed by digestive surgery.

\section{Conclusion}

This study discussed the case of a 37-year-old man diagnosed with CAPD-related tuberculous peritonitis, EPS, post-herniorrhaphy for incisional hernia indications, hypertension in therapy, inflammatory anemia (improvement), and malnutrition. The combination of clinical symptoms, radiology, and medical pathology remained the key to diagnosing tuberculous peritonitis and EPS. Prompt and precise assessment determines an effective prognosis and medical outcome. However, patients were treated for 24 days without complaints of tightness and abdominal pain. Subsequently, the patient was discharged, with category I antituberculous drug therapy and administered prednisone $40 \mathrm{mg}$ on a daily basis.

\section{References}

1. Teitelbaum I, Burkart J. Peritoneal dialysis in core curriculum in nephrology. Am J Kidney Dis 2003;42(5):1082-96.

PMid: 14582053

2. Rohit $A$, Abraham G. Peritoneal dialysis related peritonitis due to Mycobacterium spp: A case report and review literature. J Epidemiol Glob Health. 2017;6(4):243-8. https://doi. org/10.1016/j.jegh.2016.06.005 PMid:27443487

3. Burkart JM, Golper TA, Motwani S. Encapsulating Peritoneal Sclerosis in Peritoneal Dialysis Patients; 2019.

4. Tao Li PK, Sceto CC, Piraino B, Arteaga J, Fan S, Figueiredo AE, et al. ISPD peritonitis recommendations: 2016 Update on prevention and treatment. Perit Dial Int. 2016;36(5):481-508. https://doi.org/10.3747/pdi.2016.00078

\section{PMid:27282851}

5. Abraham G, Mathews M, Sekar L, Srikanth A, Sekar U, Soundarajan P. Tuberculous peritonitis in a cohort of continuous ambulatory peritoneal dialysis patients. Perit Dial Int. 2001;21(Suppl 3):S202-4. https://doi. org/10.1177/089686080102103s34

PMid:11887821

6. Rudiansyah M, Lubis L, Bandiara R, Supriyadi R, Afiatin, Gondodiputro RS, et al. Java barb fish gallbladder-induced acute kidney injury and ischemic acute hepatic failure. Kidney Int Rep. 2020;5(5):751-3. https://doi.org/10.1016/j.ekir.2020.03.014 PMid:32405599

7. Vikrant S. Tuberculosis in dialysis: Clinical spectrum and outcome from an endemic region. Hemodial Int. 2019;23(1):88-92. https:// doi.org/10.1111/hdi.12693

PMid:30289617

8. Anand U, Kumar R, Priyadarshi RN, Kumar B. Primary encapsulating peritoneal sclerosis in a tuberculosis-endemic region. JGH Open. 2019;3(4):349-52. https://doi.org/10.1002/ jgh3.12161

PMid:31406931

9. LuiSL, ChanTM, LaiKN, LoWK. Tuberculousandfungal peritonitis in patients undergoing continuous ambulatory peritoneal dialysis. Peritoneal Dialysis Int. 2007;27(Suppl 2):S263-6. https://doi.org/10.1177/089686080702702s45

PMid:17556316

10. Milburn H, Ashman N, Davies P, Doffman S, Drobniewski F, Khoo $S$, et al. Guidelines for the prevention and management of Mycobacterium tuberculosis infection and disease in adult patients with chronic kidney disease. Thorax. 2010;65(6):559-70. https://doi.org/10.1136/thx.2009.133173 PMid:20522863

11. Guideline Treatment of Tuberculosis in Renal Disease, Version 3.0. Queensland Health; 2017. Available at:: https://www.health. qld.gov.au/_data/assets/pdf_file/0024/444507/tb-guidelinerenal.pdf. [Last accessed on $2021 \mathrm{Apr} 26]$.

12. Rudiansyah M, Bandiara R, Supriyadi R, Lubis L, Kurniaatmaja E, Nur'amin HW, et al. The severe varicella zoster infection with kidney transplant patient using immunosuppressant. Int $\mathrm{J}$ Pharm Res. 2021;13(1):852-7.

13. Brooks DC, Rosen M, Chen W. Clinical Features, Diagnosis, and Prevention of Incisional Hernias; 2019.

14. Balda S, Power A, Pappalois V, Brown E. Impact of hernias on peritoneal dialysis technique survival and residual renal function. Perit Dial Int. 2013;33(6):629-34. https://doi.org/10.3747/ pdi.2012.00255

PMid:24179105 